11

\title{
Эксперимент по повышению точности передачи шкалы времени на основе метода релятивистской синхронизации
}

\author{
(ㄱ В.Ф. Фатеев, Ю.Ф. Смирнов, А.И. Жариков, Е.А. Рыбаков, Ф.Р. Смирнов Ф
}

Всероссийский научно-исследовательский институт физико-технических и радиотехнических измерений, Менделеево, Московская обл., Россия

IE-mail: frsmirnov@vniiftri.ru

Поступило в Редакцию 21 августа 2020 г.

В окончательной редакции 25 сентября 2020 г.

Принято к публикации 26 сентября 2020 г.

\begin{abstract}
Приведены результаты эксперимента по передаче шкалы времени удаленному потребителю с применением метода релятивистской синхронизации. Передача шкалы времени выполнена при помощи перевозимых квантовых часов на автомобильном шасси по дорогам федерального значения на расстояние свыше 5 тысяч километров. Полученные результаты подтверждены независимым методом сравнений шкал времени по сигналам глобальных навигационных спутниковых систем. Погрешность метода релятивистской синхронизации не превышает $150 \mathrm{ps}$, что существенно лучше, чем при использовании других методов.
\end{abstract}

Ключевые слова: релятивистская синхронизация, перевозимые квантовые часы, релятивистские эффекты, шкала времени.

DOI: 10.21883/PJTF.2021.01.50457.18526

Перевозимые квантовые часы (ПКЧ) являются одним из наиболее точных средств передачи шкал времени (ШВ) удаленным потребителям. Для повышения точности передачи ШВ с уровнем нестабильности $10^{-15}$ и выше необходима высокоточная компенсация релятивистских уходов шкалы времени ПКЧ на трассе движения [1-4]. Метод релятивистской синхронизации основан на непрерывном вычислении и компенсации указанных эффектов вдоль трассы движения путем измерения координат и скорости движения ПКЧ с помощью приемников ГЛОНАCC/GPS. Он предложен в работе [5] и испытан в реальном эксперименте [6].

Экспериментальная передача ШВ от Государственного первичного эталона единиц времени и частоты ГЭТ 1-2018 (Менделеево, Московская обл.) осуществлена ФГУП „ВНИИФТРИ““ Государственному вторичному эталону единиц времени и частоты ВЭТ 1-5 ВосточноСибирского филиала ФГУП „ВНИИФТРИ“ (Иркутск) с помощью водородных ПКЧ с относительной нестабильностью $1 \cdot 10^{-15}$. Цель эксперимента заключалась в повышении точности передачи ШВ на основе использования метода релятивистской синхронизации.

Шкалы времени эталонов $\tau_{1}$ (UTC(SU), Менделеево, квантовые часы КЧ1) и $\tau_{2}$ (UTC(Im), Иркутск, квантовые часы КЧ2) сравнивались со шкалой времени ПКЧ $\tau_{m}$ методом прямых измерений, проводимых в местах их размещения. Транспортировка ПКЧ от ФГУП „ВНИИФТРИ“ до Восточно-Сибирского филиала осуществлялась передвижной платформой на базе серийного автомобиля, оборудованного средствами автономного энергоснабжения и поддержания температурновлажностного режима. На всем протяжении маршрута движения координаты и скорость измерялись штатным приемником сигналов ГНСC JAVAD Sigma G3T из соста- ва передвижной платформы. Текущая температура в зоне размещения ПКЧ на маршруте контролировалась термогигрометром ИВА-6А-КП-Д с погрешностью $0.3^{\circ} \mathrm{C}$, магнитное поле - с помощью измерителя геомагнитных полей П3-81-01 с погрешностью 0.1 A/m.

Эксперимент проводился поэтапно.

Этап 1 - начальная калибровка, при которой шкалы $\tau_{1}(\mathrm{UTC}(\mathrm{SU}))$ и $\tau_{m}$ ПКЧ были синхронизированы с погрешностью 50 ps. Относительная начальная расстройка частоты ПКЧ относительно частоты базового эталона составила $\Delta f_{0} / f_{0}=(22.510 \pm 0.005) \cdot 10^{-15}$. Температурный коэффициент частоты, который определялся с помощью мобильной термостатированной лаборатории, размещаемой вблизи эталона ГЭТ 1-2018, составил $K_{T}=(0.430 \pm 0.005) \cdot 10^{-15}{ }^{\circ} \mathrm{C}^{-1}$. Магнитный коэффициент частоты, измеренный с помощью катушек Гельмгольца с изменением внешнего поля на $\pm 1 \mathrm{Oe}$, составил $K_{M}=(0.40 \pm 0.05) \cdot 10^{-15} \mathrm{Oe}^{-1}$.

Этап 2 - движение ПКЧ по маршруту Менделеево (Московская обл.)-Иркутск с суммарным интервалом времени транспортировки шкалы до момента сравнения шкал $\Delta \tau=114.3 \mathrm{~h}$. Маршрут движения длиной $5384.5 \mathrm{~km}$ пролегал по дорогам федерального значения, профиль высоты изменялся от +40 до $+592 \mathrm{~m}$, скорость движения не превышала $43 \mathrm{~m} / \mathrm{s}$.

В конце маршрута движения вычислены составляющие смещения шкалы ПКЧ $\tau_{m}=T_{\mathrm{PQC}}$ относительно $\tau_{1}$ : смещение, вызванное начальной расстройкой частоты $\Delta \tau_{0}=\Delta \tau \Delta f_{0} / f_{0}=9.258 \pm 0.002 \mathrm{~ns}$; смещение вследствие температурного ухода частоты $\Delta \tau_{T}=\int_{0}^{\Delta \tau} K_{T} T(\tau) d \tau=0.124 \pm 0.002 \mathrm{~ns} ;$ смещение, вы- 
званное изменением напряженности магнитного поля на трассе, $\Delta \tau_{M}=\int_{0}^{\Delta \tau} K_{M} T(\tau) d \tau=0.165 \pm 0.021 \mathrm{~ns}$.

Релятивистское смещение шкалы $\tau_{m}$ ПКЧ относительно шкалы базовых часов $\tau_{1}$ на трассе движения $\Delta \tau_{\text {rel }}$ вычислялось по формуле [5]:

$$
\begin{aligned}
& \Delta \tau_{r e l}=\frac{1}{c^{2}}\left[\varphi_{1}+\frac{1}{2} \Omega_{0}^{2}\left(x_{1}^{2}+y_{1}^{2}\right)\right] \Delta \tau \\
& -\frac{1}{c^{2}} \int_{0}^{\Delta_{\tau}}\left[\varphi_{m}+\frac{1}{2} \Omega_{0}^{2}\left(x_{m}^{2}+y_{m}^{2}\right)+\frac{1}{2} V^{2}\right] d \tau-\frac{2 \Omega_{0} S_{\nabla}}{c^{2}},
\end{aligned}
$$

где $\Omega_{0}$ - угловая скорость вращения Земли; $\varphi_{1} \varphi_{m}\left(\tau_{1}\right)-$ гравитационные потенциалы в точках размещения КЧ1 и движущихся ПКЧ; $x_{1}, y_{1}$ - координаты базовых КЧ1; $x_{m}\left(\tau_{1}\right), y_{m}\left(\tau_{1}\right), V\left(\tau_{1}\right)$ - текущие координаты и скорость ПКЧ вдоль трассы; последний член определяет эффект Саньяка за счет движения часов вдоль трассы, причем

$$
S_{\nabla}=\frac{1}{2} \int_{\tau_{1}}^{\tau_{2}}\left[x_{m}\left(\tau_{1}\right) V_{y}\left(\tau_{1}\right)-y_{m}\left(\tau_{1}\right) V_{x}\left(\tau_{1}\right)\right] d \tau_{1} .
$$

Гравитационные потенциалы в точках размещения базовых $\varphi_{1}$ и мобильных часов $\varphi_{m}$ определялись по модели потенциала гравитационного поля Земли EIGEN-6C4 [7] в виде суммы: $\varphi_{i}=\varphi_{i}^{n}+\varphi_{i}^{a n}(i=1 ; m)$, где

$$
\varphi_{i}^{n}=\left(\mu / \rho_{i}\right)\left[1-J_{2}\left(R_{e} / \rho_{i}\right) P_{2}\left(\sin \psi_{i}\right)\right]
$$

- нормальная составляющая потенциала, определяемая нулевой и второй зональной гармоникой разложения потенциала с коэффициентом $J_{2}=1.0826 \cdot 10^{-3}$; $P_{2}\left(\sin \psi_{i}\right)=\frac{3}{2} \sin ^{2} \psi_{i}-\frac{1}{2} \quad-\quad$ полином Лежандра; $\psi_{i}$ - геоцентрическая широта точки размещения часов; $\quad \mu=3.986 \cdot 10^{14} \mathrm{~m}^{3} / \mathrm{s}^{2} \quad$ геоцентрическая гравитационная постоянная; $\rho_{i}=\sqrt{x_{i}^{2}+y_{i}^{2}+z_{i}^{2}}-$ геоцентрическое расстояние точки размещения часов; $R_{e}=6.378 \cdot 10^{6} \mathrm{~m}$ - экваториальный радиус Земли;

$$
\begin{aligned}
\varphi_{i}^{a n}= & \frac{\mu}{\rho_{i}}\left[-\sum_{n=3}^{\infty} J_{n}\left(\frac{R_{e}}{\rho_{i}}\right)^{n} P_{n}\left(\sin \psi_{i}\right)+\sum_{n=2}^{\infty} \sum_{m=1}^{n}\left(\frac{R_{e}}{\rho_{i}}\right)^{n}\right. \\
& \left.\times\left(C_{n m} \cos \left(m \lambda_{i}\right)+S_{n m} \sin \left(m \lambda_{i}\right)\right) P_{n m}\left(\sin \psi_{i}\right)\right]
\end{aligned}
$$

- аномальная составляющая потенциала, включающая зональные гармоники $J_{n}$ начиная с третьей, а также секториальные и тессеральные гармоники разложения потенциала по сферическим функциям с коэффициентами $C_{n m}, S_{n m} ; P_{n m}(\sin \psi)$ - присоединенные полиномы Лежандра; $\lambda$ - долгота рассматриваемой точки.

Расчет составляющих релятивистского смещения (1) проводился на основе массива данных объемом 58969 записей текущих координат и текущей скорости ПКЧ, снимаемых с НАП ГНСС с частотой 1 раз в $5 \mathrm{~s}$, кроме случаев срыва наблюдений за спутниками, когда интервал между соседними эпохами увеличивался от 10 до $40 \mathrm{~s}$. Погрешность измерения координат составляла $2 \mathrm{~m}$, скорости $-5 \cdot 10^{-2} \mathrm{~m} / \mathrm{s}$. При расчете учитывались гармоники потенциала не выше 13-й, поскольку их коэффициенты имеют порядок $10^{-6}$ и менее. Погрешности вычисления составляющих формулы (1) для интервала движения $\Delta \tau=114.3 \mathrm{~h}$ определялись по формулам, приведенным в работе авторов [5]. В результате получено

$$
\begin{aligned}
& \Delta \tau_{m o v}=-\frac{1}{c^{2}} \int_{0}^{\Delta \tau} \varphi_{m} d \tau=-286541.0453 \pm 0.1318 \mathrm{~ns} \\
& \Delta \tau_{V}=-\frac{1}{2 c^{2}} \int_{0}^{\Delta \tau} V^{2} d \tau=-0.7538 \pm 0.003 \mathrm{~ns} \\
& \Delta \tau_{\Omega^{2}}=-\frac{\Omega_{0}^{2}}{2 c^{2}} \int_{0}^{\Delta \tau}\left(x_{m}^{2}+y_{m}^{2}\right) d \tau= \\
& -1065307.8913 \pm 0.0002 \mathrm{~ns} ; \\
& \Delta \tau_{S}=-\frac{2 \Omega_{0} S_{\nabla}}{c^{2}} \\
& =\frac{\Omega_{0}}{c^{2}} \int_{\tau_{1}}^{\tau_{2}}\left[\left(x_{m}\left(\tau_{1}\right) V_{y}\left(\tau_{1}\right)-y_{m}\left(\tau_{1}\right) V_{x}\left(\tau_{1}\right)\right] d \tau_{1}\right. \\
& =-11.8941 \pm 0.0031 \mathrm{~ns} .
\end{aligned}
$$

Первый член формулы (1) составляет

$$
\Delta \tau_{1}=\frac{1}{c^{2}}\left[\varphi_{1}+\frac{1}{2} \Omega_{0}^{2}\left(x_{1}^{2}+y_{1}^{2}\right)\right] \Delta \tau=1351848.8199 \mathrm{~ns} .
$$

Погрешность вычисления этой составляющей ввиду очень высокой точности знания координат базовых часов пренебрежимо мала (единицы пикосекунд).

Вычисленное в конце пути суммарное релятивистское смещение составило (см. рисунок):

$$
\begin{aligned}
\Delta \tau_{r e l} & =\Delta \tau_{1}+\Delta \tau_{m o v}+\Delta \tau_{\Omega^{2}}+\Delta \tau_{V}+\Delta \tau_{S} \\
& =-(12.764 \pm 0.137) \mathrm{ns} .
\end{aligned}
$$

Полный уход шкалы мобильных часов $\tau_{m}$ относительно шкалы $\tau_{1}$ на трассе равен сумме вычисленных выше составляющих $\left(\Delta \tau_{0}, \Delta \tau_{T}, \Delta \tau_{M}, \Delta \tau_{r e l}\right)$ с учетом их среднеквадратических отклонений, включая ошибку начальной синхронизации. В результате $\Delta \tau_{m}=\tau_{m}-\tau_{1}=-(3.217 \pm 0.148) \mathrm{ns} . \quad$ В конце пути все вычисленные смещения компенсировались с помощью введения в бортовую шкалу времени ПКЧ $\tau_{m}$ корректирующей поправки $\Delta \tau^{\text {cor }}=-\Delta \tau_{m}=+3.217 \mathrm{~ns}$. В результате скорректированная шкала равна $\tau_{m}^{\text {cor }}=\tau_{1} \pm 0.148 \mathrm{~ns}$. Это свидетельствует о том, 


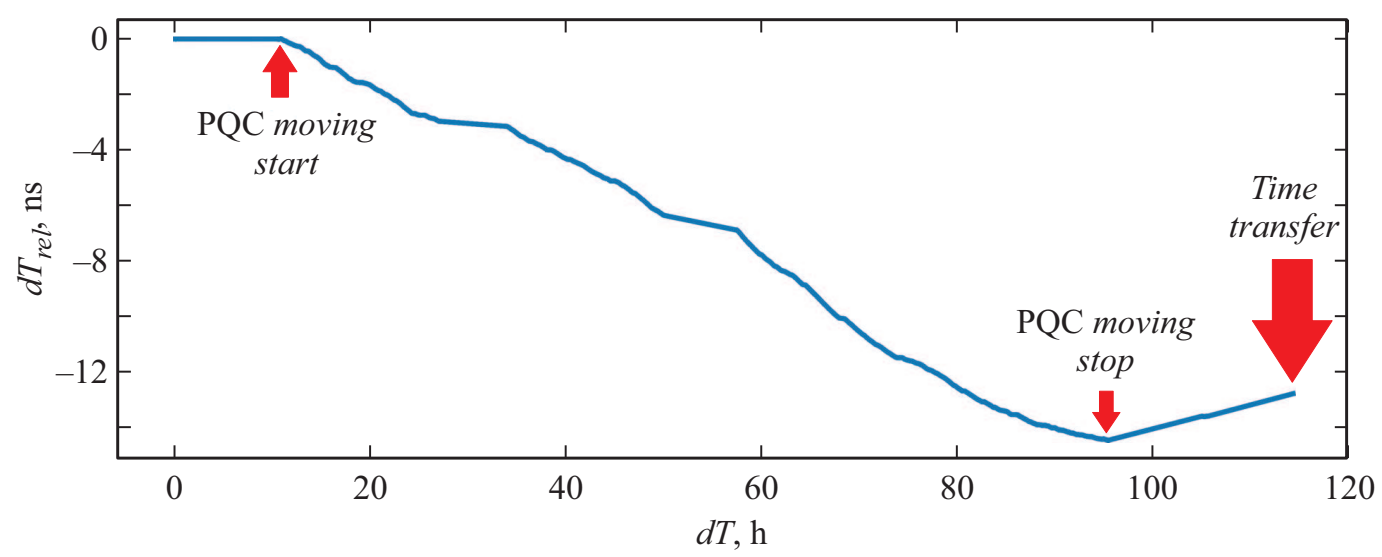

Релятивистский эффект смещения шкалы времени ПКЧ на маршруте Менделеево-Иркутск.

что ПКЧ доставили в Иркутск шкалу $\tau_{m}^{c o r}$, которая с точностью до случайной ошибки совпадает с исходной шкалой $\tau_{1}(\mathrm{UTC}(\mathrm{SU}))$.

Этап 3 - сравнение в Иркутске скорректированной бортовой шкалы времени $\tau_{m}^{c o r}=T_{\mathrm{PQC}}^{c o r}$ и синхронизируемой местной шкалы времени КЧ2 $\tau_{2}$ (UTC(Im), Иркутск). В результате разность показаний этих шкал составила

$$
\Delta \tau_{\mathrm{PQC}}=T_{\mathrm{PQC}}^{c o r}-T_{\mathrm{UTC}(\mathrm{Im})}=0.464 \pm 0.148 \mathrm{~ns} .
$$

Верификация результатов, полученных с использованием метода релятивистской синхронизации, проводилась прямым сравнением шкалы времени UTC(Im) с национальной шкалой времени $\mathrm{UTC}(\mathrm{SU})$ по сигналам ГНСC. На основе результатов сравнения независимым методом all-in-view по сигналам GPS P3 [8] установлено, что расхождение данных шкал времени на момент проведения измерений 03.09.2019 г. в 10:16 UTC составило

$$
\Delta \tau_{\mathrm{GNSS}}=T_{\mathrm{UTC}(\mathrm{SU})}-T_{\mathrm{UTC}(\mathrm{Im})}=0.6 \pm 1.5 \mathrm{~ns} .
$$

Таким образом, результат, полученный с использованием метода релятивистской синхронизации с погрешностью $0.148 \mathrm{~ns}$, находится внутри интервала неопределенности оценки расхождения шкал времени, полученной независимым методом прямых измерений по ГНСС с погрешностью $\pm 1.5 \mathrm{~ns}$.

В целом использование метода релятивистской синхронизации при передаче шкалы времени в ВосточноСибирский филиал ФГУП „ВНИИФТРИ“ на расстояние свыше 5 тысяч километров обеспечило погрешность передачи ШВ не более 150 ps. Это является новым результатом при передаче шкал времени с помощью ПКЧ на большие расстояния и обеспечивает суточную стабильность хранения шкалы времени лучше $2 \cdot 10^{-15}$.

\section{Финансирование работы}

Исследование выполнено при финансовой поддержке Российского фонда фундаментальных исследований в рамках научного проекта № 19-29-11023.

\section{Конфликт интересов}

Авторы заявляют, что у них нет конфликта интересов.

\section{Список литературы}

[1] Л.Б. Борисова, В.Н. Мельников, Измерительная техника, № 4, 13 (1988).

[2] Ю.Н. Медведев, Ю.Ф. Смирнов, в сб. Метрология времени пространства: Тр. 5-го Рос. симп. (ВНИИФТРИ, М., 1994). C. $342-343$.

[3] Б.А. Гайгеров, В.П. Сысоев, Измерительная техника, № 2, 25 (2012).

[4] В.Ф. Фатеев, В.П. Сысоев, Измерительная техника, № 8, 31 (2014).

[5] В.Ф. Фатеев, Е.А. Рыбаков, Ф.Р. Смирнов, Письма в ЖТФ, 43 (10), 3 (2017).

[6] В.Ф. Фатеев, В.П. Сысоев, в сб. Метрология времени пространства: Тр. 7-го Рос. симп. (ВНИИФТРИ, М., 2015). C. $130-144$.

[7] C. Förste, S. Bruinsma, O. Abrikosov, J.-M. Lemoine, M. Charles, J. Charles, F. Flechtner, G. Balmino, F. Barthelmes, R. Biancale, EIGEN-6C4. The latest combined global gravity field model including GOCE data up to degree and order 2190 of GFZ Potsdam and GRGS Toulouse. GFZ Data Services (2014). https://doi.org/10.5880/icgem.2015.1

[8] Бюллетень Е-09-2019/Е5. Государственный вторичный эталон единиц времени и частоты ВЭТ 1-5.

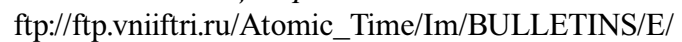
2019/be0919-5 